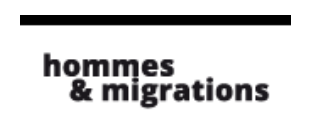

\section{Hommes \& migrations}

Revue française de référence sur les dynamiques

migratoires

Musulmanes et féministes en Grande-Bretagne

\title{
Un œil pour mémoire
}

Usages, valeurs et réinterprétations du bijou traditionnel chez les héritiers de l'exil maghrébin

\section{Slimane Touhami}

\section{(2) OpenEdition}

Journals

Édition électronique

URL : http://journals.openedition.org/hommesmigrations/1858

DOI : 10.4000/hommesmigrations.1858

ISSN : 2262-3353

Éditeur

Musée national de l'histoire de l'immigration

Édition imprimée

Date de publication : 1 septembre 2012

Pagination : 105-112

ISSN : 1142-852X

Référence électronique

Slimane Touhami, «Un œil pour mémoire », Hommes \& migrations [En ligne], 1299 | 2012, mis en ligne le 29 mai 2013, consulté le 22 septembre 2020. URL : http://journals.openedition.org/ hommesmigrations/1858; DOI : https://doi.org/10.4000/hommesmigrations.1858

Ce document a été généré automatiquement le 22 septembre 2020.

Tous droits réservés 


\title{
Un œil pour mémoire
}

\author{
Usages, valeurs et réinterprétations du bijou traditionnel chez les \\ héritiers de l'exil maghrébin
}

\section{Slimane Touhami}

1 Pour les héritiers de l'exil maghrébin, le legs culturel prend place à différents niveaux. Il se manifeste dans l'usage plus ou moins maittrisé de la langue, et dans l'attachement à une confession, à des rites et à des codes transmis dans le cercle familial. Il constitue l'étoffe du lien, à la fois physique et sentimental, entretenu avec le bled, repère structurant pour des jeunes en quête de légitimité. Il prend encore forme dans l'artefact exposé dans l'espace public qu'est le bijou façonné selon les canons de la tradition. La petite main qui se balance au bout d'un collier signe l'héritage. Elle a valeur de marqueur qui dit les origines mais qui raconte, en même temps, d'autres histoires. Support aux émotions suscitées par le souvenir de l'être aimé ou générées par l'ombre du sort, le signe est aussi le reflet de valeurs et d'idéologies qui délimitent un rapport au monde marqué du sceau de la singularité. En affichant un récit sur l'intime mais également sur la société, l'objet témoigne d'une trajectoire individuelle qui sinue entre les références culturelles.

2 En invitant le lecteur à arpenter l'espace du sens chez les enfants d'un Maghreb revisité, l'article projettera un rai de compréhension sur la relation entretenue avec les significations culturelles envisagées comme don. À partir des données d'une enquête ethnographique menée entre 2005 et 2007 auprès de jeunes dans les quartiers de relégation toulousains - les grands ensembles du Mirail et d'Arnaud-Bernard, lieux de la présence maghrébine dans le centre-ville -, il s'agira, en décodant les motivations et les circonstances qui prévalent au port du bijou, de montrer comment, derrière l'usage commun, la référence à la forme traduit un discours sur soi et également sur autrui. Une approche - l'exercice d'une ethnographie du quotidien et de la dérive à l'affût du détail - qui correspond à un projet : valoriser le retour au réel à l'ère du spectacle en éprouvant, à l'échelle de la peau, la complexité d'une jeunesse qui se révèle plus que jamais éclatée en un faisceau d'expériences contrastées. 


\section{Parades}

3 À la sortie des lycées ou dans une rame de métro, l'œil peut noter la présence, à même le corps, d'une marque dont les racines plongent de l'autre côté de la mer. Le bijou traditionnel chez les jeunes se décline sous trois formes qui composent la triade d'une sémiotique de l'exil. L'“œil bleu", pour reprendre les termes usités par les informateurs, est un petit disque de verre teinté sur fond d'or, très apprécié ces dernières années. Il concurrence la khemsa, ou main de Fatma, petite main d'or et filigrane qui voit sa popularité décliner depuis vingt ans. Dans le sillage du retour du religieux, ayat el korsi - le verset 256 de la seconde sourate du Coran - marque des points en s'affichant au cou des jeunes, ses caractères sacrés se découpant dans le métal le plus précieux. Ces trois bijoux sont les plus observés à Toulouse, loin devant le fer à cheval ou le yazz, symbole d'une berbèrité revendiquée.

Dans cette géographie du symbole, le même l'emporte pourtant sur le différent. Un format, une matière donnent corps au signifiant. Garçons et filles portent le bijou en pendentif, enfilé au bout d'un collier de chaîne ou de mailles. L'or, en alliage ou plaqué, est la référence en termes de métal précieux. Surtout, une fonction commune relie des signes aux histoires divergentes. L'œil, la main et la lettre sont d'abord envisagés comme des remparts contre l'invisible. Cette fonction apotrope vise une calamité redoutée pour ses effets sur les plus fragiles. CEuvre de "l'envieux qui envie" selon la formule coranique ${ }^{1}$, l'aïn, le mauvais œil en Afrique du Nord, est le maléfice engendré par la nefs, le moi profond, qui se distingue de cette autre forme spirituelle avec laquelle il cohabite, le rouh', le souffle vital d'origine divine ${ }^{2}$. Empruntant les chemins du regard et de la parole, en particulier la louange dont le miel cache à la vue le fiel de l'envie, l'œil - car tel est son nom - s'en prend à l'objet du désir. Les plus jeunes sont particulièrement exposés. Étant objets d'admiration, ils peuvent s'attirer un mal qui maltraite ses victimes à coups de crises de pleurs, de maux de tête violents et d'amaigrissement soudain avec, parfois, la mort en guise de dénouement. Ainsi, au Maroc, l'œil de l'étranger, mais aussi celui, plus inquiétant, de l'ami ou du parent incapable de maîtriser ses pulsions, sont accusés de remplir les deux tiers du cimetière.

\section{Passer la main}

5 C'est pour parer à cette menace que plusieurs informateurs se sont vu offrir un bijou dans les premiers mois de leur vie. Affaire de famille, ce don est fait par les femmes de la proche parenté: mère, grand-mère, tante ou sœur. Dans les propos, elles apparaissent au premier plan de la lutte contre le mal, intervenant auprès des plus petits pour délivrer le bijou-cuirasse. Ce souci de garantir le plus vulnérable de l'envie d'autrui, l'œil ethnographique le consigne sur le terrain en notant la présence, sur le corps des enfants, d'une armure d'or et d'arabesques passée dans une épingle à nourrice fixée sur les langes ou pendue à un petit collier.

6 Cet usage prophylactique est maîtrisé par la majorité des jeunes interrogés. Si celles et ceux qui ont reçu le signe lors de leur petite enfance revendiquent un attachement sentimental au bijou - c'est le cas de Mounia, 28 ans, très liée à la khemsa offerte à sa naissance : "Je ne peux pas m'en séparer (...). Ça me rappelle ma mère" -, la principale motivation qui gouverne au port du signe reste la protection contre le mal. Pour les jeunes nés sous l'ombre du malheur, l"aïn, cette "poisse", expression, selon les termes 
recueillis, de la "mauvaise langue" qui médit ou de la "grande bouche qui parle trop", est l'objet d'un crédit important. À l'âge adulte, le sort change d'oripeaux pour être responsable de l'échec à l'examen, de l'accident de voiture ou de la perte d'un emploi. Dans cette situation, le bijou ne cesse de faire barrage comme au premier jour. Pour Rachid, chauffeur-livreur de 25 ans dont la souche familiale se trouve dans l'Ouest algérien ${ }^{3}$, l'“œil" reçu à l'enfance joue toujours son rôle, exposé sur la poitrine, "contre les gens qui parlent trop. Les hypocrites et les jaloux".

7 Si les premières années de la vie inaugurent une relation durable avec le signe, il est possible de s'armer ultérieurement. Les vacances estivales au bled sont l'occasion de s'équiper - ou de se rééquiper - en défenses symboliques. Amulettes et talismans sont là-bas disponibles dans des espaces urbains dédiés au métal jaune - les "marchés de l'or" ou souks dahab - qui sont visités à dessein tant pour la finesse de leurs productions que pour des prix défiant toute concurrence.

Il n'est pourtant pas besoin de passer la mer pour acquérir le signe. À Toulouse, les commerces dits "bazars" proposent aussi, sur leurs étals, des objets bon marché mais de qualité moindre, fabriqués en Chine ou au Pakistan. Des bijouteries spécialisées dans la clientèle maghrébine présentent, pour leur part, des productions de facture supérieure. Elles sont, pour partie, tenues par des juifs rapatriés d'Afrique du Nord qui perpétuent, avec l'orfèvrerie, une activité ancestrale.

\section{Effets de mode}

9 Né en Algérie, Monsieur M. tient une bijouterie près du Capitole. Le samedi, jour d'affluence, la boutique est occupée par des clientes affairées qui, d'un œil averti, commentent la qualité d'un diadème, examinent ne bague ou simplement regardent, l'air songeur, les trésors exposés dans la devanture.

Les amulettes occupent ici une place de choix. Khemsas, étoiles de David et fers à cheval sont disposés en damier sur le présentoir de suède vert : "On m'achète pas mal de mains. C'est à cause des superstitions." "Superstitions" qui, précise Monsieur M., sont communes aux juifs et aux musulmans: "Le mauvais ceil, on y croit (...). C'est comme la main, juifs et Arabes se la partagent. On a tellement vécu longtemps ensemble qu'à force, on a fini par adopter les mêmes choses."

11 Pourtant, c'est à un autre symbole que revient aujourd'hui la faveur de la clientèle : "Il y a une demande importante pour l'œil bleu. C'est ce qui marche le mieux ces derniers temps." La mode est à la petite goutte de verre teinté lovée sur un fond d'or ou de brillants. Rare il y a encore vingt ans, elle a désormais la préférence de ceux qui cherchent une garantie contre le mal. Pour cette Marocaine rencontrée dans la boutique, la chose est entendue : "Mes enfants ne jurent que sur ça."

Dans le Maghreb d'antan, le symbole oculaire a pris place parmi les meilleures réponses contre l'aïn. L'œil, qui renvoie le mal à la face du fascinateur, est représenté sur les tatouages faciaux, les tapis et jusque sur les gandouras, les lourds manteaux de laine des montagnards.

13 Pourtant, les actuelles reproductions en pâte de verre ne s'inscrivent pas dans une tradition nord-africaine ${ }^{4}$. Leur aire initiale est à rechercher plutôt vers le bassin oriental de la Méditerranée 5 . En Grèce, les reproductions d'yeux bleus se rencontrent à l'entrée des maisons, entre autres. En Turquie, le nazar boncuq reste la manifestation la 
plus ostentatoire d'une croyance au mauvais œil fortement ancrée en Anatolie : rares sont ceux qui n'arborent pas dans leur automobile, pendu au rétroviseur d'intérieur, un signe qui s'apparente peut-être à l'antique oudjat - l'œil d'Horus -, une amulette très populaire dans l'Égypte ancienne utilisée contre la fascination et les paroles d'envie ${ }^{6}$. Revisité par la mondialisation - l'objet est produit en série dans des pays comme l'Italie, la Tunisie, voire la Chine -, "l'œil bleu" témoigne de l'influence de l'Ailleurs sur l'art de contrer l'œil envieux au Maghreb comme dans ses diasporas.

C'est que cet exilé a pour lui de nombreux atouts. Outre sa qualité de nouveau venu qui attire la curiosité, il bénéficie d'une réputation d'efficacité. Ainsi Mme Mimouna, propriétaire d'un petit commerce dans un quartier populaire de la Ville rose, a-t-elle décidé de se mettre à la page en choisissant, elle aussi, "l'œil bleu. Il paraît que c'est bon contre l"aïn. Je l'ai mis au cou de mes enfants." Le discours portant sur le pouvoir de l'amulette se transmet de bouche à oreille, le meilleur moyen de garantir sa diffusion : “ l'œil bleu en or, c'est bien d'après ce que disent les gens", affirme Sihem, une étudiante qui a succombé au bijou lors de ses dernières vacances au Maroc. L'aspect agréable de la petite perle azur cerclée d'or a également motivé son choix : "En plus, c'est mignon. Ça se porte avec tout." Khadija, une jeune employée de bureau, avance un même argument esthétique qui compose avec l'usage préventif initial : "Personnellement, je trouve ça beau, discret."

15 Autre avantage et non des moindres, l'œil bleu est envisagé comme une alternative à la khemsa, la main de Fatma, déconsidérée ces dernières années avec le retour d'un discours rigoriste. Pour Khadija, "l'œil bleu, c'est mieux que la main. C'est haram - illicite - à ce qu'on dit." Sihem utilise la même rhétorique. Ainsi son bijou est-il plus acceptable que "la khemsa. Il y a des gens qui te disent que ça, c'est païen, contraire à l'islam. Si tu regardes bien, ça se tient. La main de Fatma, on croit que c'est musulman mais on n'en parle nulle part dans le Coran. Ça n'a rien de religieux."

\section{Textes coraniques}

16 Avec la popularisation des thèses orthodoxes en matière religieuse, la khemsa tend, en effet, à être perçue comme une manifestation du shirk, l'idôlatrie, source de déviance en islam. La forme, dont les racines se perdent dans les temps anciens de la jahiliya, le paganisme préislamique, se serait greffée en périphérie de la Loi, détournant les croyants de la voie originelle. Ce discours est patent chez les tenants du verset du Trône, ayat el korsi. Sous ce nom se cache le verset 256 de la seconde sourate. Sa traduction par Blachère donne: "Allah - nulle divinité exceptée Lui - est le Vivant, le Sublime. Ni somnolence ni sommeil ne Le prennent. A Lui ce qui est dans les cieux et ce qui est sur la terre. Quel est celui qui intercédera auprès de Lui, sinon sur Sa permission? Il sait ce qui est entre les mains des Hommes et derrière eux, alors qu'ils n'embrassent de Sa science, que ce qu'Il veut. Son Trône s'étend sur les cieux et la terre. Le conserver ne Le fait point ployer. Il est l'Auguste, l'Immense?."

17 Ce verset possède une fonction talismanique importante. C'est une panacée utilisée contre différentes formes de dangers. On le récite avant un voyage ou face à tout sentiment de perdition ${ }^{8}$. Il est aussi réputé efficace contre le regard envieux. L'usage au quotidien du passage coranique détermine un large éventail d'objets qui captent le texte sur différents supports. Porte-clés et autocollants fixent la lettre porteuse de bienfaits. "L"'aïn, c'est véridique (...). C'est dans le Coran. Mais porter la main, non. Les 
personnes qui la portent sont dans l'erreur (...). Pour celui qui veut se protéger, il y a des sourates et la ayat. C'est tout ${ }^{9}$." Les propos de Khalid, un ouvrier de 27 ans qui porte discrètement le talisman confirment cette idée, partagée par de nombreux jeunes, qui veut que seul le Texte fasse loi en terme de défense contre l'Invisible. Emprunter la voie de la rectitude suppose, de fait, le rejet de pratiques religieuses appréhendées comme des apports extérieurs à l'islam.

Cette volonté de revisiter la religion en expurgeant de sa substance des éléments suspects est presque aussi ancienne que la naissance de l'Ultime Révélation. Au VIII ${ }^{\mathrm{e}}$ siècle déjà, Abd el-Wahhab, un juriste originaire du Nadjd, dans l'actuelle Arabie Saoudite, dénonce les superstitions et les innovations qui se sont introduites dans l'islam de la région ${ }^{10}$. Plus tard, dans l'Algérie coloniale, le mouvement des oulémas dénoncera l'usage des amulettes et les visites aux saints au prétexte qu'ils égarent le croyant $^{11}$.

Cette quête de la voie droite conduit des adeptes de la lettre à s'interdire l'appui du bijou. Ainsi Fatima, une jeune femme d'une trentaine d'années très impliquée dans les associations de son quartier situé dans la couronne des grands ensembles. Dans la salle du petit local où se déroule la rencontre, elle définit sa conception de la protection fondée sur le Texte: "Contre le mauvais ceil, il n'y a rien de mieux que le Coran. Tu as des livres avec des ayats - des adjurations - pour soigner les maladies. On peut aussi faire des du'a des invocations ${ }^{12}$." Aucune mention, par contre, de l'objet en métal précieux. "Après, tout ce qui est bijou, machin, personnellement, j'évite. Pour moi, c'est de l'artifice, ces pendentifs-là, tous ces trucs où tu retrouves des extraits du Coran (...).Normalement, il faut éviter l'or, surtout pour les hommes (...). C'est écrit dans les textes." Fatima fait référence aux hadiths, ces récits traditionnels qui consignent les paroles et les actes attribués au Prophète. Plusieurs passages du Sahih Al Muslim et Bukhari ou du Kitab as Sunnan de Abou Dawud, compilations de hadiths qui font autorité dans l'islam sunnite, prohibent en effet chez l'homme le port de matières de valeur comme l'or ou la soie ${ }^{13}$.

\section{Réinventions}

20 Rejetée par les religieux, la khemsa subit aussi l'accusation du bord opposé. Ainsi, les débats autour de la loi de 2004 sur les signes religieux à l'école ont été un prétexte à une charge contre l'amulette. Arguant du principe de laïcité, l'ancien président de la République Jacques Chirac a, par exemple, assimilé, dans un discours officiel inaugurant le projet de loi, la main de Fatma à un signe confessionnel à bannir des salles de classe ${ }^{14}$.

Désapprouvée de part et d'autre, l'amulette, qui n'est reliée à l'islam que par une dénomination désuète, a fini par perdre sa faveur auprès des Maghrébins de France. L'aura de celle qui ne serait rien d'autre que l'héritière de la main de Tanit, charme carthaginois dont la mémoire locale aurait gardé le souvenir, a décliné sous le coup des critiques. Ce désintérêt pour la khemsa coïncide avec une redéfinition de sa charge symbolique chez les générations nées en France. En effet, si la plupart des jeunes interrogés connaissent l'usage traditionnel de l'amulette, d'autres, lui adjoignent de nouvelles significations. Hamid, un jeune homme d'une trentaine d'années dont la famille est originaire des hauts plateaux oranais, voit par exemple dans la khemsa un indicateur de l'identité confessionnelle, faisant sienne l'interprétation exogène 
dominante: "En fait, tu vois tous ces jeunes qui font la fête. Ils boivent, ils fument et en plus, ils portent la main de Fatma. C'est un signe religieux mais ça ne les empêche pas de faire tout ça."

Il arrive que les acteurs se tournent vers les explications du "dehors" pour donner sens à leurs pratiques, les valeurs nouvelles venant occuper les cases vacantes d'une transmission familiale. C'est ce que démontrent encore Ali, 32 ans, rencontré un matin de février 2004 dans un café du quartier Arnaud-Bernard. Pour lui, la khemsa sert à dire la culture dont on estime être le légataire ${ }^{15}$ : "La main (...) c'est quelque chose qui signifie notre identité, notre culture à nous, les Maghrébins (...) Même chose avec les Catalans qui mettent un âne derrière leur voiture ou les Bretons avec l'autocollant BZH." La khemsa peut être, en effet, conçue comme l'emblème de l'être maghrébin. Cette conception, on la repère dans l'iconographie actuelle. Lorsque l'on fait figurer la main de Fatma sur un tee-shirt ou une affiche pour un festival de raï, on fait référence non pas au symbole protecteur ni à celui désignant une confession religieuse, mais bel et bien à ce qui est devenu, au fil des années, le blason d'un espace culturel qui se décline aussi dans l'Ailleurs.

23 Témoin des préoccupations identitaires chez les descendants d'un exil postcolonial, le signe le plus ancien s'avère être le plus ouvert. Ouverture au sens mais aussi au goût des Autres. Quelques heures d'observation dans n'importe quelle ville française suffisent à reconnaître le caractère non exclusif d'un bijou qui compense, par sa popularité auprès des non-musulmans, la perte d'estime dans son espace premier. En cela, la petite main tant décriée fait lien entre les mondes, dressant une passerelle pardessus des lignes de partage que d'aucuns, aujourd'hui, considèrent comme des frontières hermétiques et figées.

\section{La peau en question}

Cette traversée de l'espace du sens chez une jeunesse française met en lumière la vivacité d'une expérience religieuse à l'ombre des mondes urbains. Le signe matérialise la relation à une croyance - celle qui fait la part belle à l'œil envieux - qui prend place en exil non sur le principe d'une réplication du même mais en se reconfigurant selon les normes dominantes. Histoires de mauvais œil et histoires de vie. Car la pièce d'orfèvrerie rend visible, aussi, une trajectoire personnelle qui joue à la fois sur le mode de l'intime, en éclairant le rapport à l'univers parental et à l'identité, et sur le dehors, en traduisant un regard sur le monde qui articule valeurs et idéologies. Derrière le masque de la tradition s'expriment des sensibilités et des points de vue souvent divergents, parfois opposés, qui soulignent la diversité d'une présence aux racines elles-mêmes plurielles. Répertorier différents regards dans le rapport à soi et aux autres reste ainsi redevable d'une ethnographie qui scrute l'élémentaire et l'accessoire tapi dans les plis du contemporain. La traque d'un diable dont on dit qu'il affectionne les détails permet d'interroger les appartenances et les allégeances, les croyances et les idéologies d'une époque marquée par la refonte des modèles préexistants et l'innovation. La mobilisation de l'œil ethnographique, qui cible ici un corps réinvesti en ces temps de doutes, représente l'une des voies pour questionner une modernité qui se réinvente chaque jour, se nouant et se dénouant à partir de ce film qui fait support à l'intime mais sert aussi de frontière et de bannière, à savoir la peau. 


\section{NOTES}

1. Coran, CXIII-5.

2. Coran, L-15.

3. Toulouse accueille depuis les années soixante une diaspora algérienne originaire du nordouest du pays. Ainsi, si Paris est associé à l'exil kabyle et Marseille à l'Oranie, Toulouse reste la ville d'accueil des habitants de Mostaganem et de son arrière-pays.

4. Edward Westermarck, Survivances païennes dans la religion mahométane, Payot, Paris, 1935, p. 65.

5. Dominique Champault, Amand Raymond Verbrugge, "La main. Ses figurations au Maghreb et au Levant." in Le Catalogue du Musée de l'Homme- série B- Afrique blanche et Levant, s-e, Paris, 1965, p. 12.

6. Gaston Maspero, L'Archéologie égyptienne, Librairies-imprimeries réunies, Paris,1887, p. 235.

7. Coran, II-56.

8. Malek Chebel, Dictionnaire des symboles musulmans. Rites, mystique et civilisation, Albin Michel, Paris,1995, art. "Trône".

9. D'autres passages du Coran sont employés contre l'aïn à titre prophylactique ou en cure. C'est le cas des sourates dites de l'Unité de Dieu (CXII), de l'Aube (LXXXIX) ou la Fatiha (I)

10. Louis Gardet, Cheikh Bouamrane, Panorama de la pensée islamique, Sindbad, Paris, 1984, p. 165.

11. Jean-Paul Charnay, La Vie musulmane en Algérie d'après la jurisprudence de la première moitié du $\mathrm{XX}^{\mathrm{e}}$ siècle, Presses universitaires de France, Paris, 1991, p. 66.

12. Allusion aux tibb ennebi, les "médecines du Prophète". Publiés en français, ces corpus d'indications thérapeutiques tirées du Coran et des hadiths servent de base à la Roqya, l'art de se soigner selon un modèle prophétique.

13. "(Le Prophète) nous a interdit de porter des bagues en or, de boire dans des vases en argent, de se servir des selles en soie" (Sahih Al Muslim, n 5129) ; "Le Prophète d'Allah a pris de la soie et l'a tenu dans sa main droite et a pris de l'or et l'a tenu dans sa main gauche et a dit: les deux d'entre ceux-ci sont interdits aux hommes de ma communauté" (Kitab as Sunnan, $n^{\circ}$ 4046). Textes in University of Southern California,Compendium of MuslimTexts : http://www.usc.edu/schools/ college/crcc/engagement/resources/texts/

14. Sophie Bessis, "Pauvre main de Fatma!", Le Monde, 16 janvier 2004.

15. David Le Breton, Signes d'identité. Tatouages, piercings et autres marques corporelles, Paris, Métaillé, 2002, p. 19.

\section{RÉSUMÉS}

Les enfants reçoivent en héritage de leurs parents tout un ensemble de pratiques culturelles, de croyances et d'objets. En contexte post-colonial, les Maghrébins de France entretiennent des rapports particuliers avec ce don qu'est le pendentif destiné à parer les attaques du mauvais œil, l'aïn, source de maux et de mort. Significations religieuses, relation au monde des parents et à la société dominante s'entremêlent pour légitimer son port. Chez de jeunes Toulousais, ces signes constituent autant un indicateur du changement culturel qu'un reflet des visions du monde d'une jeunesse qui s'expérimente dans la diversité. 
AUTEUR

SLIMANE TOUHAMI

Docteur de l'EHESS Laboratoire LISST-Toulouse 2 